HRJ v.2 n.12 (2021)

Recebido: 21/06/2021

Aceito: 29/07/2021

\title{
Reflexões da Psicologia sobre o Câncer de Mama: Uma Cartilha Informativa
}

\author{
Alliny Michelly Vale ${ }^{1}$ \\ Laís Lopes Barcelos ${ }^{2}$ \\ Milena Lima Santos ${ }^{3}$ \\ Renata Costa Fortes ${ }^{4}$
}

1. Psicóloga Residente do Programa Multiprofissional em Atenção ao Câncer da Escola Superior de Ciências da Saúde - ESCS/FEPECS.

2. Psicóloga Residente do Programa Multiprofissional em Atenção ao Câncer da Escola Superior de Ciências da Saúde - ESCS/FEPECS.

3. Psicóloga Residente do Programa Multiprofissional em Atenção ao Câncer da Escola Superior de Ciências da Saúde - ESCS/FEPECS.

4. Nutricionista, Doutora e Mestra em Nutrição Humana, Coordenadora do Programa Multiprofissional em Atenção ao Câncer da Escola Superior de Ciências da Saúde - ESCS/FEPECS

Email para correspondência: fuh.vale@gmail.com

\section{RESUMO}

Introdução: O câncer de mama é definido pelo crescimento desordenado e incontrolável de células anormais no tecido mamário. Objetivo: Disseminar o conhecimento acerca dos impactos psicológicos da neoplasia mamária para a população, através da educação em saúde. Método: Realizado um estudo exploratório, sendo os dados obtidos através das principais bases de dados: a plataforma online do Instituto Nacional de Câncer (INCA), o Ministério da Saúde, o Oncoguia, o Plano Oncológico do Distrito Federal, artigos e capítulos de livros de Psico-Oncologia referentes ao sofrimento psíquico da mulher diagnosticada com câncer de mama. A execução da cartilha foi realizada pelo editor gráfico Canva. Resultados e Discussão: Produção de cartilha informativa, contendo os principais achados da literatura: definição, aspectos epidemiológicos, fatores de risco, prevenção, sinais e sintomas, diagnóstico, tratamentos, direitos das pacientes, rede de atenção oncológica do Distrito Federal e considerações da Psicologia. Conclusões: O câncer de mama é uma doença ameaçadora de vida. Nesse sentido, o acompanhamento psicológico é de extrema importância para o manejo em todas as fases do adoecimento.

Palavras-Chave: Neoplasias da mama, Psico-oncologia e Educação em saúde

\section{Psychology's Reflections about Breast Cancer: An Information Booklet}

\author{
ABSTRACT \\ Introduction: Breast cancer is defined by the disordered and uncontrollable growth of \\ abnormal cells in the breast tissue. Objective: To disseminate knowledge about the
}


psychological impacts of breast cancer for the population through health education. Methods: An exploratory study was carried out, with data obtained from the main databases: the online platform of the Instituto Nacional de Câncer (INCA), the Ministério da Saúde, Oncoguia, the Plano Oncológico do Distrito Federal, articles and book chapters of PsychoOncology regarding the psychological distress of women diagnosed with breast cancer. The execution of the booklet was carried out by the graphic editor Canva. Results and Discussion: Production of an informative booklet containing the main findings in the literature: definition, epidemiological aspects, risk factors, prevention, signs and symptoms, diagnosis, treatments, patients' rights, oncology care network of the Federal District and Psychological considerations. Conclusions: Breast cancer is a life threatening disease. In this sense, psychological support is extremely important for the management at all stages of the illness,

Keywords: Breast Neoplasms, Health Education, Psycho-Oncology

\section{INTRODUÇÃO}

O câncer de mama é definido pelo crescimento desordenado e incontrolável de células anormais no tecido mamário. Câncer é o nome genérico para um grupo heterogêneo de doenças com manifestações clínicas distintas e, portanto, com sintomatologia e terapêuticas variadas ${ }^{1}$. O tipo histológico mais comum para o câncer de mama feminina é o carcinoma de células epiteliais, que se divide em lesões in situ e invasoras ${ }^{1}$.

No mundo, o câncer de mama é considerado o câncer mais incidente entre as mulheres. No Brasil, estimam-se 66.280 novos casos de câncer de mama, para cada ano do triênio 2020-20221.

De acordo com dados publicados pelo Instituto Nacional do Câncer (INCA), a taxa de mortalidade por câncer de mama é a primeira causa de morte por câncer na população feminina brasileira, com 14,23 óbitos/100.000 mulheres em 2019. As regiões Sudeste e Sul são as que apresentam as maiores taxas, com 16,14 e 15,08 óbitos/100.000 mulheres em 2019, respectivamente ${ }^{1}$

Importante destacar que, a incidência do câncer de mama tende a crescer progressivamente a partir dos 40 anos, assim como a mortalidade por essa neoplasia ${ }^{1}$. 
Nesse sentido, é importante compreender que não existe um único fator de risco para o desenvolvimento do câncer de mama. A literatura aponta como principais ${ }^{2}$, o envelhecimento (a partir dos 50 anos de idade), história familiar (câncer de mama ou ovário), alterações genéticas (mutações dos genes BRCA1 e BRCA2), estilo de vida (sedentarismo, alimentação inadequada, uso de bebida alcoólica e obesidade), exposição a radiações ionizantes, nuliparidade, menopausa tardia e reposição hormonal pós-menopausa.

Os principais sinais e sintomas ${ }^{2}$ da doença são o aparecimento de nódulos (geralmente indolores e duros); alterações nos mamilos; manchas avermelhadas na mama ou parecidas com "casca de laranja"; linfonodos na axila e secreções anormais.

Praticar atividade física, manter uma alimentação saudável, manter o peso corporal adequado e evitar o uso de bebidas alcoólicas e cigarro são formas de prevenir o câncer, inclusive o câncer de mama.

Ao perceber alterações corporais, a literatura aponta para a importância da busca por avaliação médica especializada em uma Unidade Básica de Saúde (UBS). Destaca-se aqui, a importância do autoexame² e da mamografia para o rastreio da doença (mulheres de 50 a 69 anos a cada dois anos) $)^{2}$.

Os principais tratamentos para o câncer de mama são cirurgia, quimioterapia, radioterapia, hormonioterapia, imunoterapia, cuidados paliativos e reabilitação ${ }^{3}$.

Existem direitos sociais direcionados a pacientes com diagnóstico de doença oncológica $^{4}$, como a Lei dos 60 dias (12.732), que assegura o acesso ao tratamento em até sessenta dias após o diagnóstico. Esses direitos são conquistas importantes que visam assegurar a assistência integral aos pacientes diante do adoecimento e tratamento.

Nesse sentido, é possível destacar alguns direitos voltados para os pacientes com câncer de mama, a exemplo, a Lei 12.802, que assegura cirurgias plásticas de reconstrução às mulheres que foram submetidas à cirurgia para a retirada total ou parcial da mama em função 
do câncer. Além disso, existe a Lei da Mamografia (lei 11.664) que garante a realização de mamografia a todas as mulheres, a partir dos 40 anos de idade 5 .

A Política Nacional de Prevenção e Controle do Câncer (Portaria 874/2013) estabelece que a assistência em saúde deva ser regionalizada e descentralizada, partindo da noção de território. A rede de atenção oncológica do Distrito Federal é formada pela atenção básica, secundária, terciária e quaternária, de acordo com o seu grau de complexidade e investimento tecnológico. Toda a rede deve oferecer assistência especializada e integral ao paciente com câncer, atuando na promoção, proteção, diagnóstico, estadiamento, tratamento e reabilitação ${ }^{3}$.

Apesar de raro, faz-se necessário salientar que os homens também podem desenvolver câncer de mama. Sua abordagem em ensaios clínicos e publicações é pouco frequente, corresponde a apenas $1 \%$ de todos os cânceres mamários. Os fatores de risco incluem hiperestrogenismo, idade, ascendência judaica, história familiar e síndrome de Klinefelter. O tratamento é semelhante ao do câncer de mama na mulher: cirurgia, hormonioterapia, quimioterapia e radioterapia. Seu prognóstico varia conforme a extensão do tumor e grau de invasão. Dessa forma, ressalta-se mais uma vez a importância do diagnóstico precoce ${ }^{6}$.

Embasado no referencial teórico citado, o objetivo desta cartilha informativa é disseminar o conhecimento acerca dos impactos psicológicos da neoplasia mamária para a população, por meio da educação em saúde.

\section{MÉTODOS}

Para a construção da cartilha informativa foi realizado um estudo exploratório por meio de uma revisão da literatura, promovendo o embasamento teórico para o desenvolvimento da produção científica. Os dados foram obtidos através das principais bases 
científicas acerca do câncer de mama: a plataforma online do Instituto Nacional de Câncer (INCA), do Ministério da Saúde, do Oncoguia e o Plano Oncológico do Distrito Federal.

Para o embasamento teórico da Psicologia foram analisados artigos e capítulos de livros da Psico-Oncologia referentes ao sofrimento psíquico da mulher diagnosticada com câncer de mama.

A cartilha informativa foi realizada através do programa Canva, um editor gráfico gratuito, que permite a criação de designs personalizados. O layout da cartilha é de autoria própria.

\section{RESULTADOS}

Produção de cartilha informativa, contendo os principais achados da literatura: definição, aspectos epidemiológicos, fatores de risco, prevenção, sinais e sintomas, diagnóstico, tratamentos, direitos das pacientes, rede de atenção oncológica no Distrito Federal e considerações da Psicologia. 


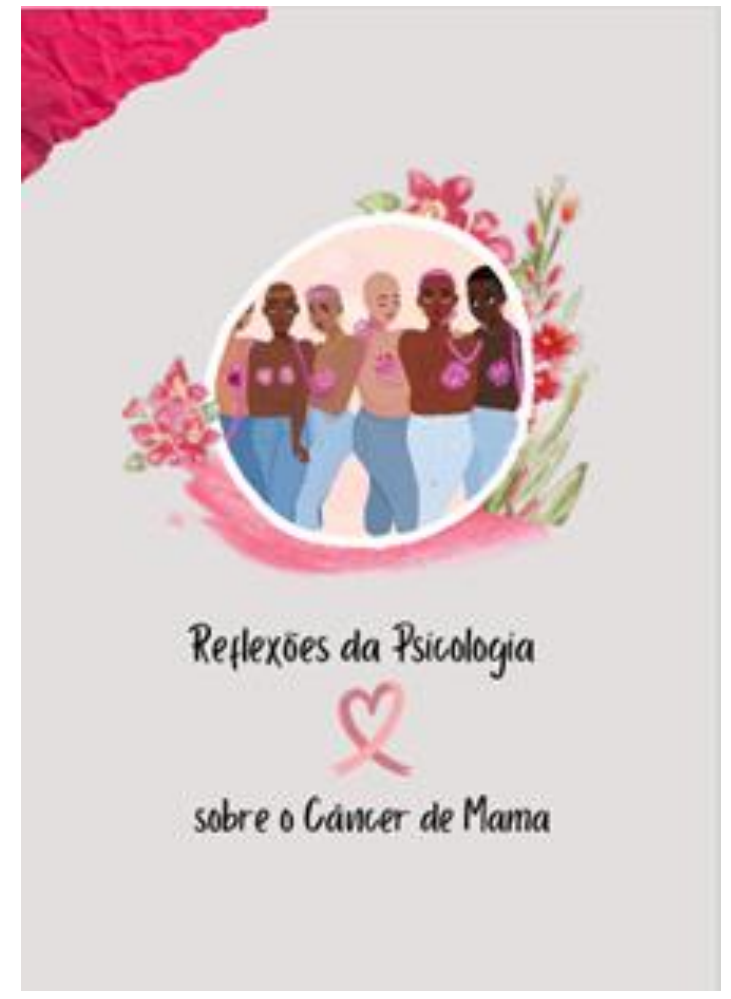

\section{Autoras}

Nis vale

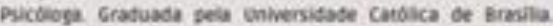

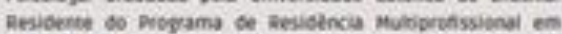

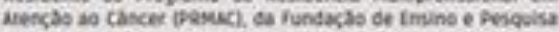
en cilncias di saose verics, vinculads a secrecarts de sade. os Distrino federal ises on

Las topes sarcelos

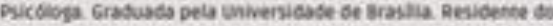

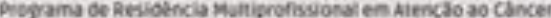

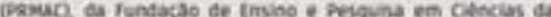

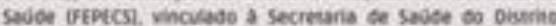
Federaitses on?

Finens uims obs sanes

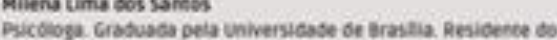

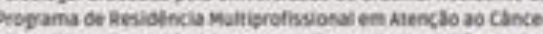

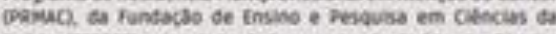

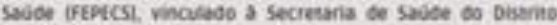
feceraikses on

Orsentadere

Reente cesta fortes

herestacosta lortes

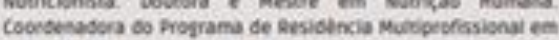

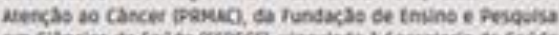
en Cilncias da saude otwecs, vinculado a secrearia de Sader oo biserito federatcesson.
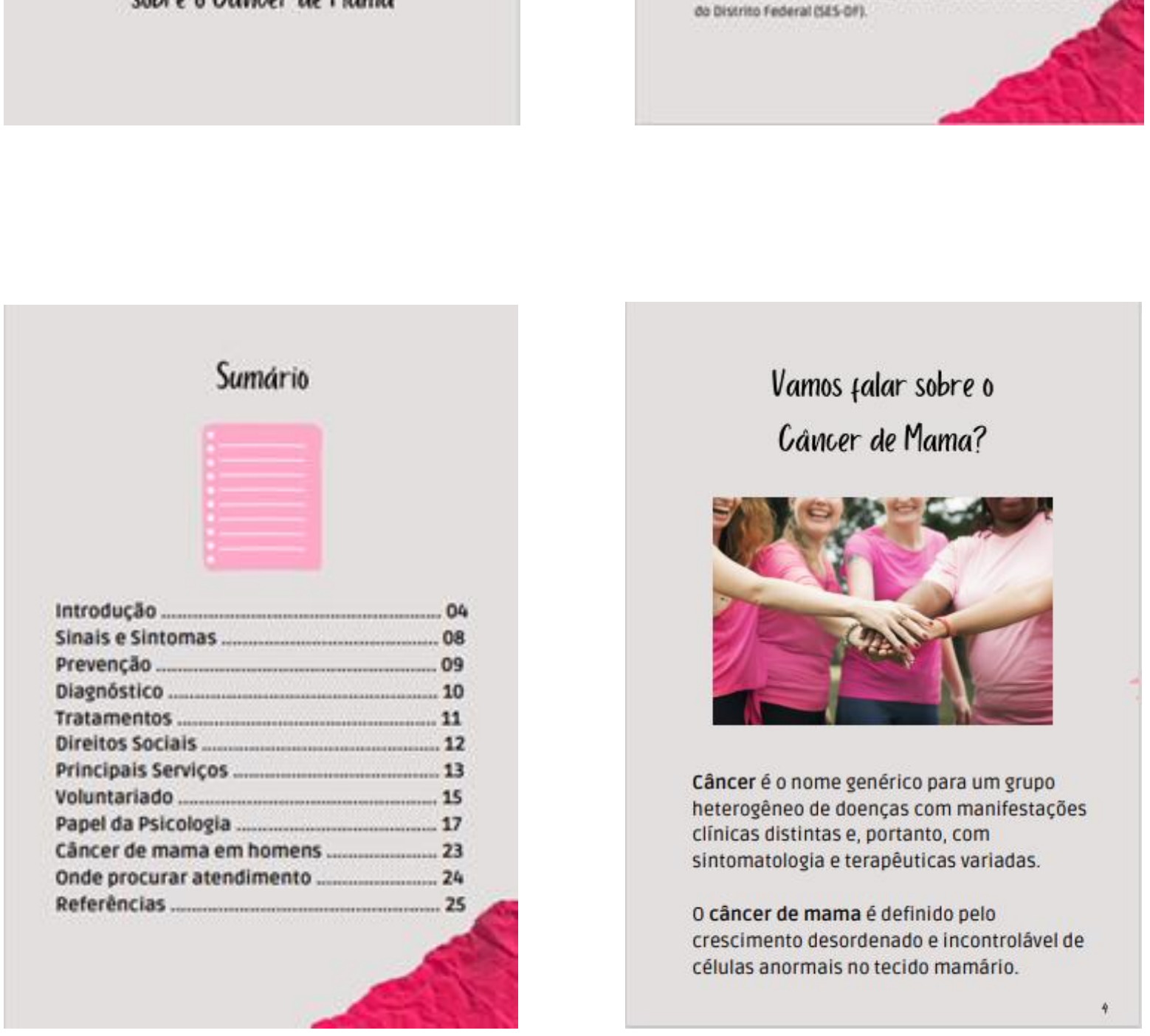
No mundo, 0 câncer de mama é considerado 0 câncer mais incidente entre as mulheres. No Brasil, estimam-se 66.280 novos casos de câncer de mama, para cada ano do triênio 2020-2022.

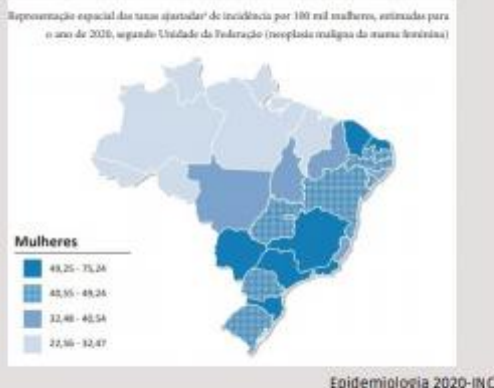

De acordo com o Instituto Nacional do Câncer (INCA), a taxa de mortalidade por câncer de mama ajustada pela população mundial representa a primeira causa de morte por câncer na população feminina brasileira, com 14,23 óbitos/100.000 mulheres em 2019.
Importante destacar que, a incidência do câncer de mama tende a crescer progressivamente a partir dos 40 anos, assim como a mortalidade por essa neoplasia.

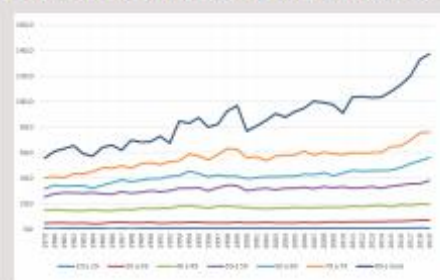

Taxa de mortalicade par Cancer de Mama Feminina, especiticas per faixas etarias, por 100000 mulheres, Brasili, 1979 a 2019, INCA

Não existe um único fator de risco para o

desenvolvimento do

câncer de mama. A seguir estão os principais.

\section{0 que causa o Cancer de} Mama?

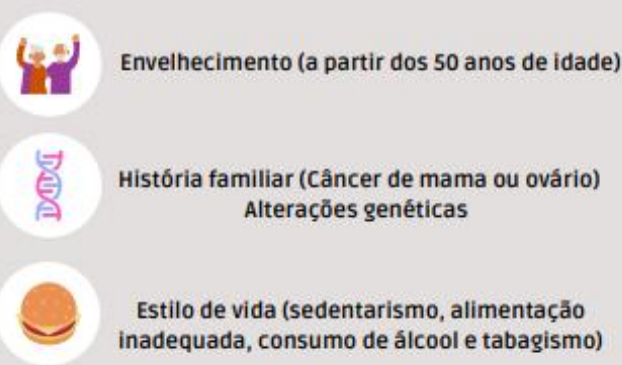

Exposição a radiaçōes ionizantes

Menopausa tardia

Reposiçăo hormonal

Nuliparidade

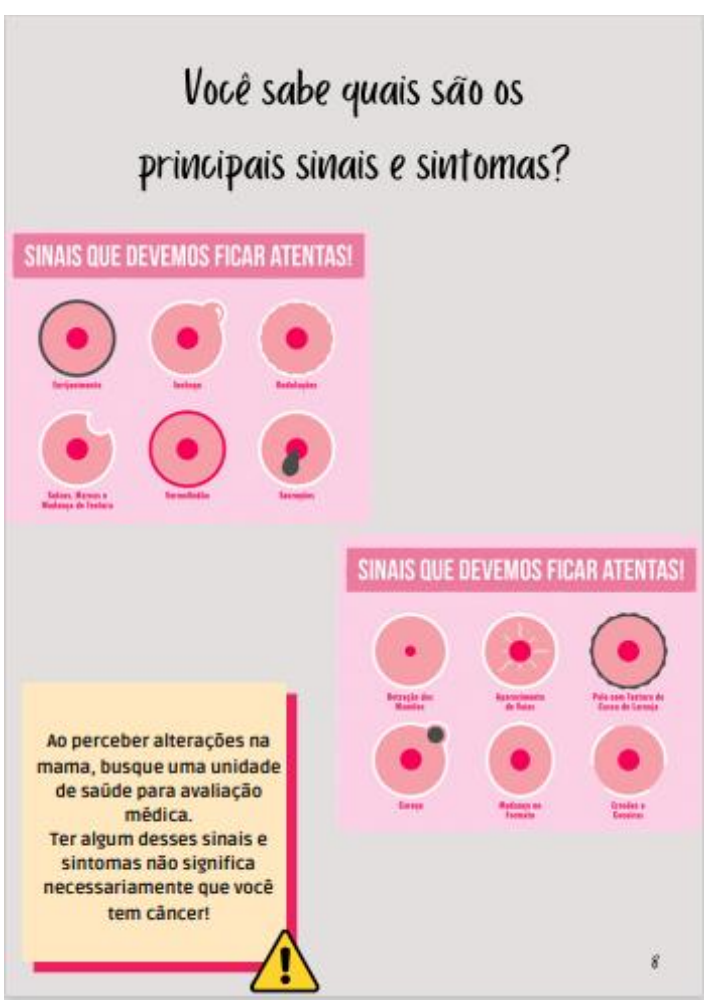




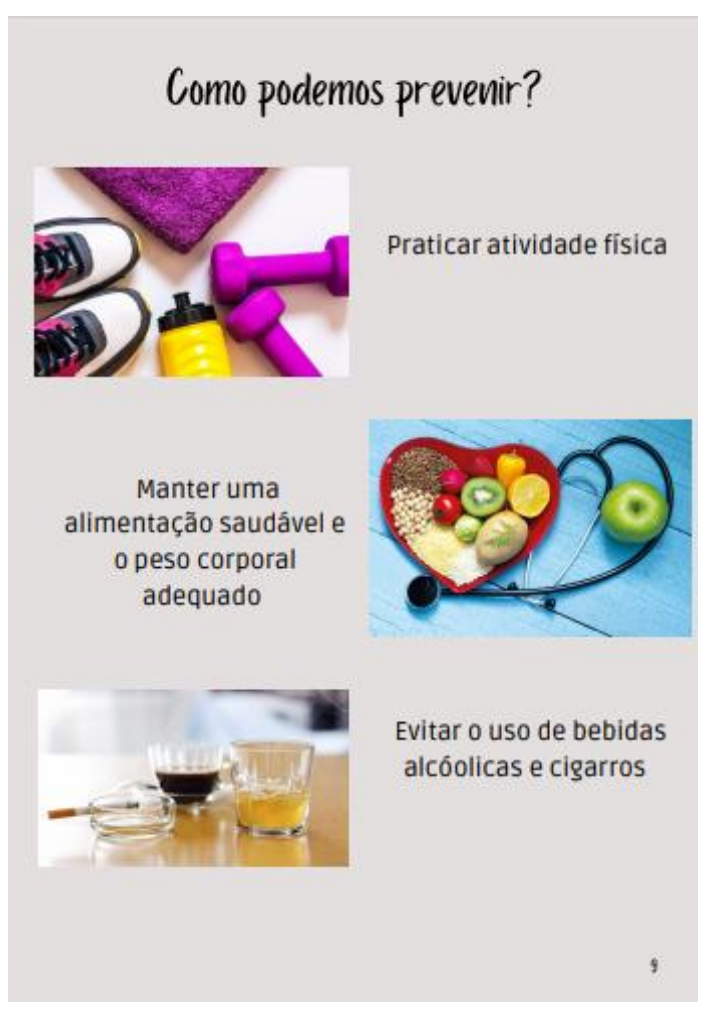

\section{Ecomo é feito o diagnóstico?}

- Através da mamografia, que é uma radiografia das mamas, capaz de visualizar alteraçōes suspeitas.

- Através do exame clínico, que consiste na observação e a palpação das mamas por um médico ou enfermeiro.

Quem deve fazer a mamografia para rastreio da doença?

0 exame é recomendado para

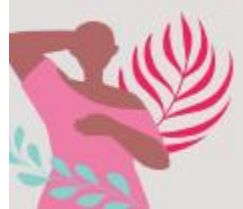
mulheres de 50 a 69 anos a cada dois anos.

\section{Quais são os tratamentos?}

Cirurgia;

Quimioterapia adjuvante ou neoadjuvante;

Radioterapia;

Hormonioterapia;

Imunoterapia;

Cuidados paliativos;

Reabilitação.

\section{Quais são os direitos das mulheres} com Cancer de Mama?

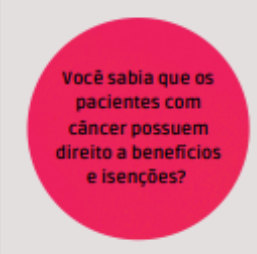

A seguir os principais direitos da mulher com câncer de mama:

Cirurgia Reconstrutiva Mamária (lei 12.802)

Para as mulheres que realizaram cirurgia para a retirada total ou parcial da mama em função do cânce o SUS realiza cirurgias plásticas de reconstrução.

Lei da mamografia (lei 11.664)

o suS assegura a realização de mamografia a todas as mulheres, a partir dos 40 anos.

Lei dos 60 dias (12.732)

$\checkmark$ A lei assegura que a paciente tenha direito a receber tratamento em até 60 dias após o diagnóstico de câncer.

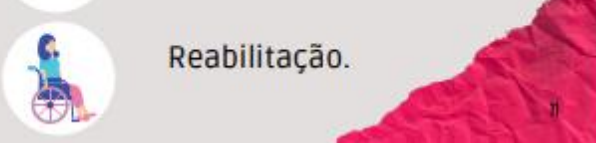

Para saber de todos os direitos, acesse: hetps://shortest.link/et: 


\section{Vamos conhecer a rede de} a tençẫo ao câncer do DF?

Hospital de Apoio de Brasília (Cacon);

- Hospital de Base do Distrito Federal (Cacon);

Hospital Regional da Asa Norte (Cacon);

Hospital Regional de Ceilândia (Cacon);

Hospital Regional do Gama (Cacon);

- Hospital Regional de Sobradinho (Cacon);

Hospital Regional de Taguatinga (Cacon);

- Hospital Sarah /Associaçăo đas Pioneiras Sociais (Unacon);

- Hospital Universitário de Brasília/Fundaçăo da Universidade de Brasilia (Unacon).

\section{Como conseguir \\ atendimento?}

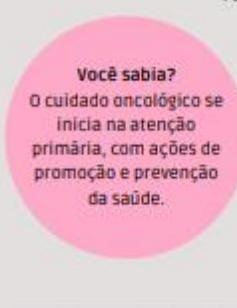

Conheça como funciona a linha de

cuidado para pacientes com

câncer:

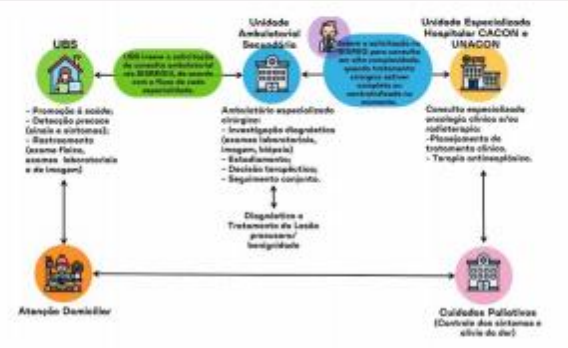

\section{Onde buscar ajuda?}

\section{Rede Feminina de Combate ao Câncer}

oficina de prótese mamária e lenços;

Doação de cabelos e confecção de perucas;

Distribuição de cestas básicas, itens de higiene, roupas e lenços:

Transporte solidário;

Lanche solidário;

Campanhas de educação em saúde;

- Casa Rosa (Casa de apoio para pacientes oncológicos de outros estados);

Eventos sociais com música e arte.

(D) Hospital de Base do Distrito Federal S.M.H.S - Area especial, quadra 101, Asa Sul, Brasîia-DF/CEP: 70390 090. Fone: (61) 3364-5467/ 3550-8900 - Ramal: 9178 (sala de is acolhimento); Ramal: 9277 (sala de acolhimento).

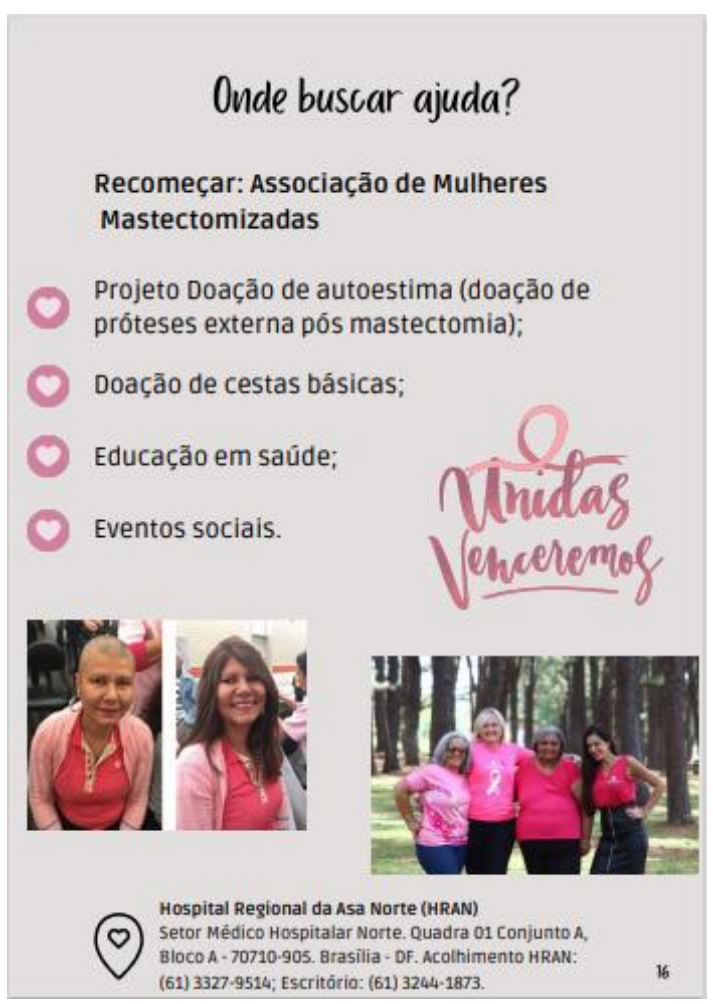



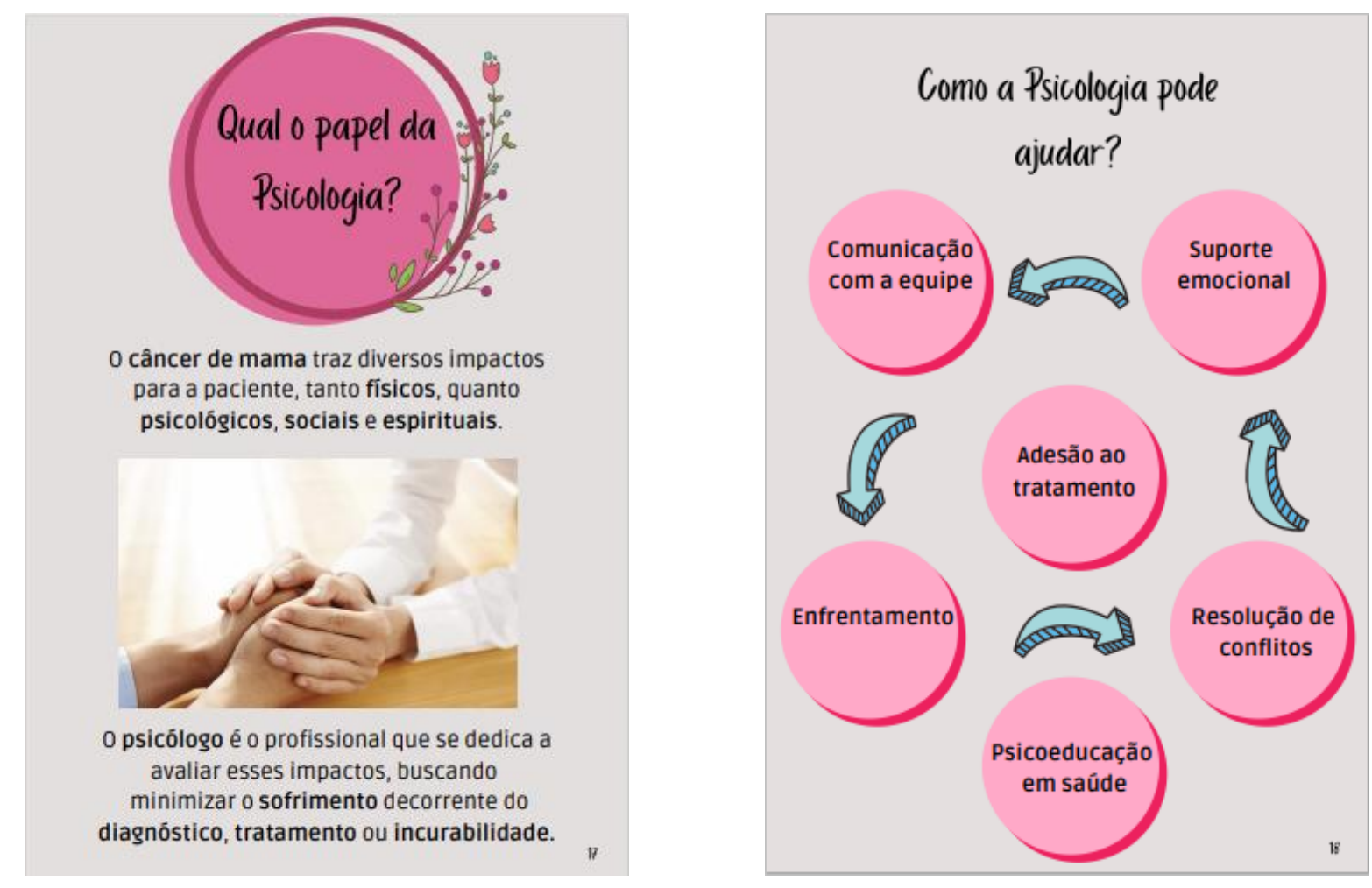

\section{Aspectos psicológicos do \\ Cancer de Mama}

A atuação da Psicologia no câncer de mama busca compreender 0 significado da doença para cada mulher e as mudanças decorrentes do diagnóstico e do tratamento.

Sentimentos comuns:

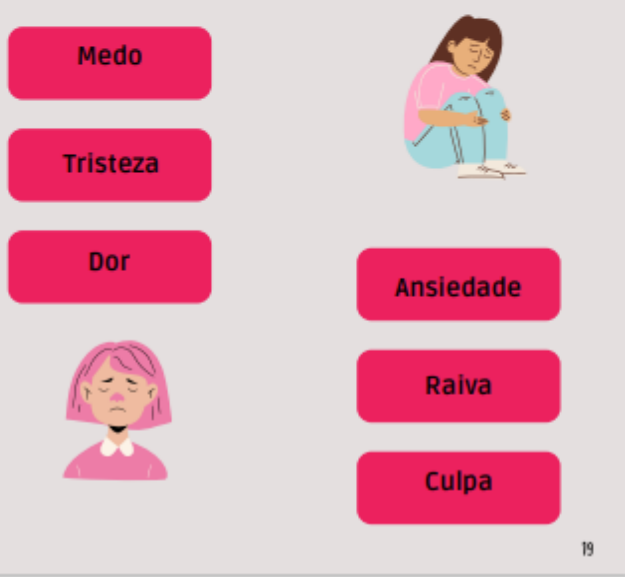

\section{Aspectos psicológicos do \\ Cancer de Mama}

Demandas comuns:

Mudanças nas relaçōes interpessoais, questōes relacionadas à sexualidade, conflitos conjugais, baixa autoestima, sofrimento em relação à mutilação do corpo, ganho ou perda de peso, fadiga crônica, perda do cabelo, sentimento de não ser mais uma mulher completa e possivel infertilidade. Mesmo após o término do tratamento, muitas referem medo de recidiva, de metástases e da morte.

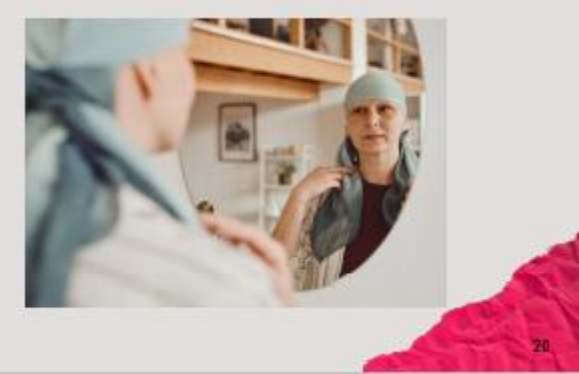




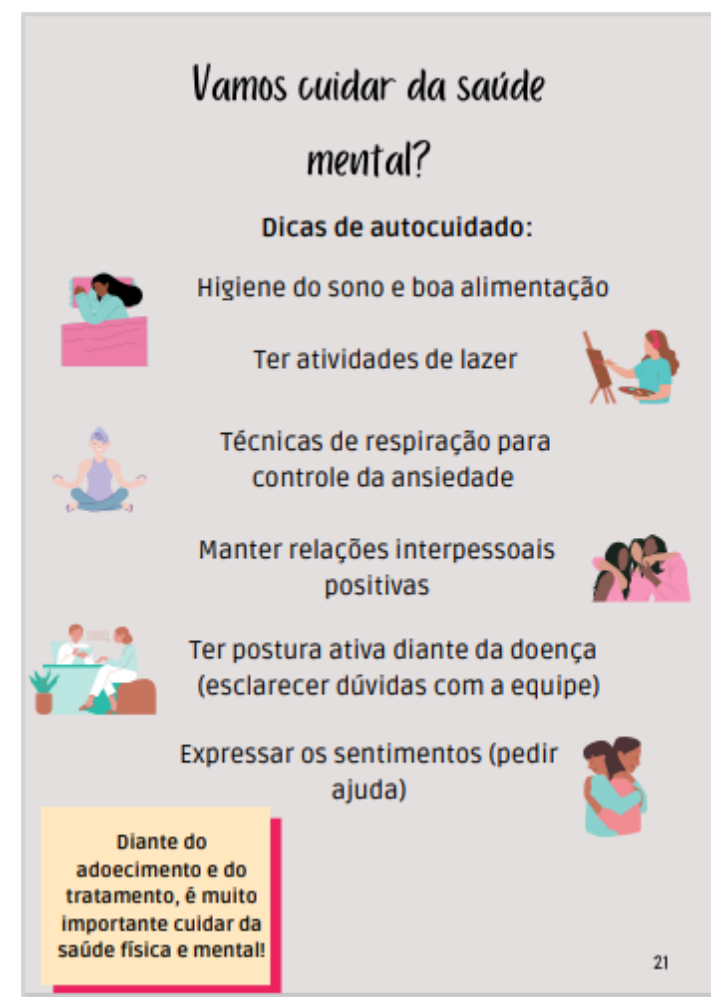

\section{Vamos cuidar da saúde mental?}

Modalidades de atendimento:

0 acompanhamento psicológico individual é indicado ao longo de todo o processo de adoecimento.

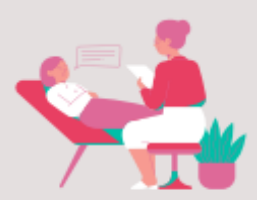

A terapia de casal é um recurso para expressão de sentimentos e construçăo de estratégias saudáveis para lidar com a doença $e$ promover a comunicaçăo.

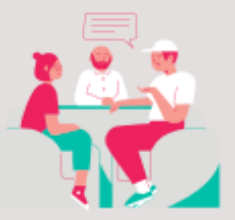

A terapia de grupo, é um recurso potente para expressão emocional por meio da identificação entre os participantes.

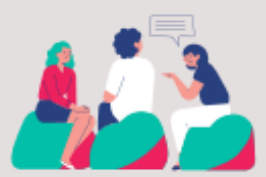

\section{Você sabia?}

0 câncer de mama em homens é uma doença pouco conhecida. Corresponde a apenas $1 \%$ de todos os cânceres mamários. Os fatores de risco incluem hiperestrogenismo, idade, ascendência judaica, história familiar e síndrome de Klinefelter. 0 tratamento é semelhante ao do câncer de mama na mulher: cirurgia, hormonioterapia, quimioterapia e radioterapia. Seu prognóstico varia conforme a extensão do tumor e grau de invasão. Dessa forma, ressaltase mais uma vez a importância do diagnóstico precoce.

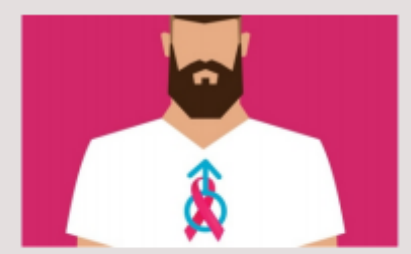

\section{Locais para atendimento}

\begin{tabular}{ll} 
PSicológico & $\begin{array}{c}\text { vocẽ sabia? } \\
\text { Devido à pandemia, } \\
\text { alģuns desses } \\
\text { serviços estâo } \\
\text { atendendo online. }\end{array}$ \\
\hline Clínica-Escolas: & Uniceub-Asa Sul - (61) 3966-1626;
\end{tabular}

- IESB - Asa Sul e Ceilândia - (61) 3445-4502 / (61) 3962-4748;

- UCB - Taguatinga - (61) 3356-9328 / (61) 3356-9000;

UnB - Asa Norte - (61) 3107-1680;

Uniceplac - Gama - (61) 3035-3957;

\section{Outros Serviços - Saúde Mental:}

- CAPS - Centro de Atenção Psicossocial - referenciado de acordo com seu território;

- Hospital São Vicente de Paula - Taguatinga Sul - (61) 2017-1450 (3612/3613);

- ISM - Instituto de Saúde Mental - Riacho Fundo - (61) 3399 3666 / 2017-1900 (7710/7711); 


\section{Referências}

Aguiar, M. A F. et al (org.). PSICO-onCoLogia: caminhos de cuidado. Så Paulo: Summus, 2019. 396-403.

Câncer de mama é preciso falar disso. Disponivel em: <http://busms.saude.gov.br/bus/publicacoes/cancer_mama_prec iso_falar_disso.pdf>, acesso em abril de 2021.

C. conheça os direitos das mulheres em relaçăo ao câncer de mama. Disponivel em:

<http://woww.oncoguia.org.br/conteudo/conheca-os-direitos-dasmulheres-em-relacao-a0-cancer-de-mama/13006/42/> acesso em abril de 2021

(1) Controle do cancer de mama. Disponivel em: <https://Www. inca.gov.br/controle-do-cancer-de-mama/conceitoe-magnitude>, acesso em abril de 2021.

(1) Nogueira, S. P., Mendonça, J. V. D., \& Pasqualette, H. A. P. (2014). Căncer de mama em homens. Rev Bras Mastologia, 24(4), 109-114.

(1) Recomeçar: Associaçăo de Mulheres Mastectomizadas. Disponivel em: <https://www. recomecar.orgl?, acesso em abril de 2021.

Q Rede Feminina de Combate ao Cancer. Disponivel em: <https://Www.redefemininabrasilia.org.br/>, acesso em abril de 2021.

(9) Secretaria de Estado de Saúde đo Distrito federal. Plano oncologico. Disponivel em: <http://www saude.df.gov.br/wpconteudo/uploads/2018/02/Plano-oncologico-formatadofinal.pdf $>$, acesso em abril de 2021

\section{Referências}

SILVA, L. C.. Câncer de mama e sofrimento psicológico: aspectos relacionados ao feminino. Psicol. estud., Maringá , v. 13, n. 2, p. 231-237, Junho, 2008

(1) Silva, L. C. D. (2008). Câncer de mama e sofrimento psicológico: aspectos relacionados ao feminino. Psicologia em estudo, 13(2), 231-237.

Tipos de câncer de mama. Disponível em: <https://WWW.nationalbreastcancer.org/types-of-breastcancer/ $>$, acesso em abril de 2021

(1) Tipos de câncer đe mama. Disponivel em: <https://WWW.nationalbreastcancer.org/types-of-breastcancer/>, acesso em abril de 2021

(1) Tudo sobre os direitos dos pacientes com câncer de mama. Disponivel em: $<$

https://fundacaolacorosa.com/wp-content/uploads/2020/01/7udosobre-os-direitos-dos-pacientes-com-c\%c3\%A2ncer-de-mama-1.pdfs acessado em abril de 2021

(1) Venâncio, J. L. (2004). Importância da atuaçăo đo psicólogo no tratamento de mulheres com câncer de mama. Revista brasileira de cancerologia, 50(1), 55-63.

(1) Vinogradov, S., \& Yalom, 1., D. (1992). Manual de psicoterapia đe grupo.

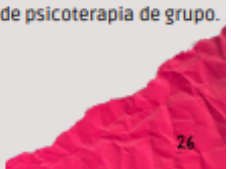

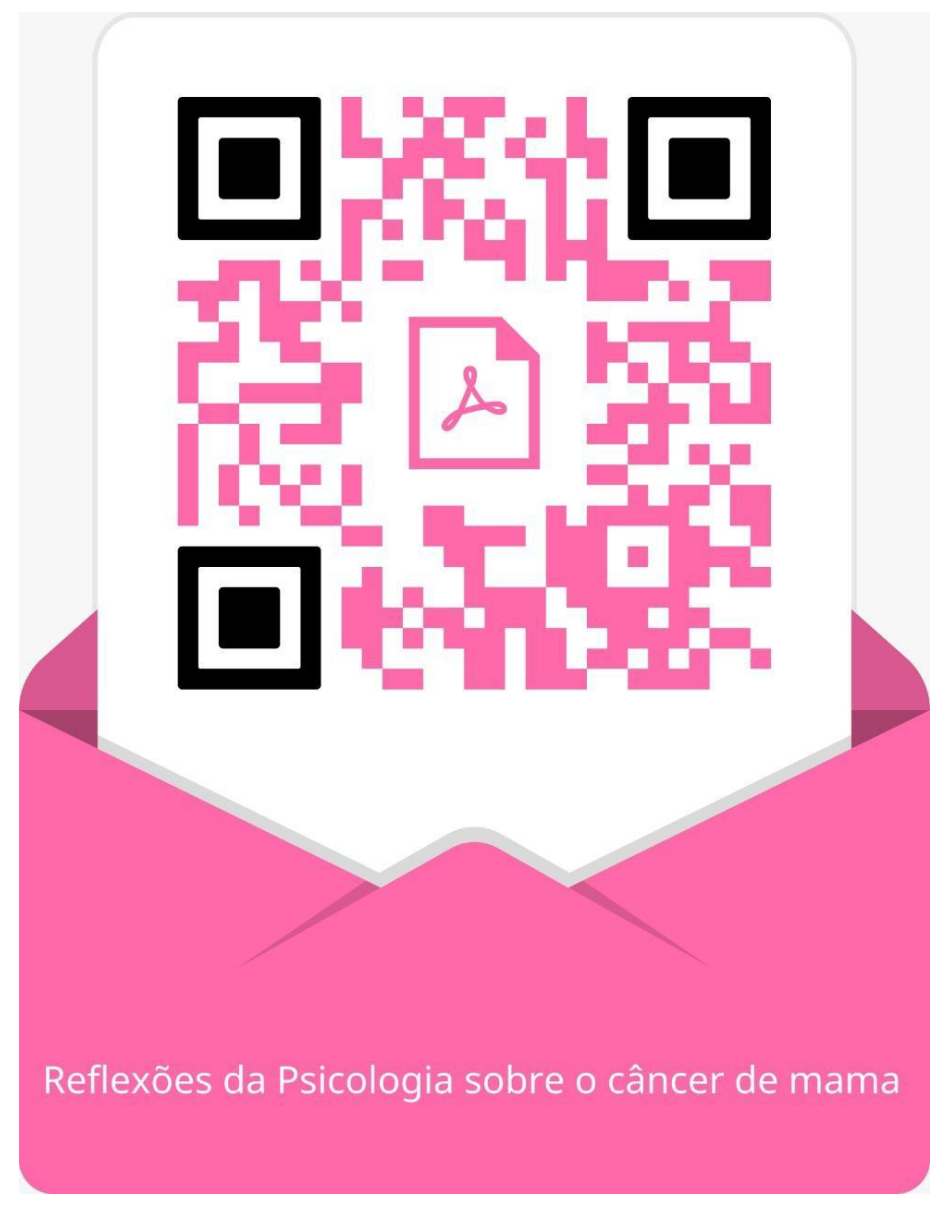




\section{DISCUSSÃO}

O câncer de mama, assim como os demais tipos de câncer, traz diversos impactos para a paciente, tanto físicos, quanto psicológicos, sociais e espirituais. O psicólogo é o profissional que se dedica a avaliar esses impactos, buscando estratégias para minimizar o sofrimento decorrente do diagnóstico, tratamento ou impossibilidade de cura ${ }^{7}$.

A Psicologia auxilia na adesão ao tratamento, favorece a construção de estratégias adaptativas e funcionais para lidar com o adoecimento, facilita a comunicação do paciente com a equipe, auxilia na resolução de conflitos, realiza a psicoeducação em saúde e oferece suporte emocional para os pacientes e familiares ${ }^{7}$.

A atuação da Psicologia no câncer de mama possui suas especificidades. É necessário compreender o significado do adoecimento para cada mulher e quais foram as mudanças decorrentes do diagnóstico e do tratamento. Muitas mulheres compartilham sentimentos de tristeza, ansiedade, medo, dor e raiva. Relatam também mudanças nos relacionamentos interpessoais, dificuldades relacionadas à sexualidade, conflitos conjugais, baixa autoestima, sofrimento em relação à mutilação do corpo, ganho ou perda de peso, fadiga crônica, perda do cabelo, sentimento de não ser mais uma mulher completa e possível infertilidade. Mesmo após o término do tratamento, muitas referem medo de recidiva, de metástases e da morte ${ }^{8}$.

O acompanhamento psicológico é de extrema importância para intervir nas demandas que causam sofrimento tanto para a paciente quanto para a sua rede de apoio (familiares, amigos, por exemplo). O impacto psicossocial do câncer de mama na mulher reflete em sua identidade e percepção simbólica do feminino, podendo gerar diminuição da autoestima e medo da rejeição 9 .

No que diz respeito à sexualidade, considerando a importância das mamas na construção social da feminilidade, o enfrentamento de uma enfermidade nessa parte do corpo impõe à mulher mudanças na prática sexual, no desejo, no prazer, na aceitação do corpo, na 
autoimagem, na sensação de segurança e bem-estar consigo mesma. A mulher sente dificuldade de expressar sua intimidade e de se adaptar à nova identidade 9 .

O acompanhamento psicológico individual é indicado ao longo de todo o processo de adoecimento, desde a fase anterior ao diagnóstico, ou seja, desde o momento de percepção dos primeiros sintomas, até a fase de alta, devido ao medo da recidiva, ou fase de fim de vida, considerando o trabalho com foco no conforto para conviver com a doença ${ }^{10}$.

A terapia de casal é um recurso para expressão de sentimentos e construção de estratégias saudáveis para lidar com o adoecimento e promover a comunicação entre os casais, possibilitando maior conexão e acolhimento do sofrimento ${ }^{10}$.

Já a terapia de grupo é um recurso potente para expressão emocional por meio da identificação entre os participantes. Existem diversas possibilidades de práticas grupais ao pensarmos em pacientes com diagnóstico de câncer de mama, podendo ser citados: grupos com mulheres com diagnóstico recente; grupos para pacientes em Cuidados Paliativos; grupos de sala de espera para pacientes em tratamento quimioterápico e grupos para familiares de pacientes com câncer de mama ${ }^{11}$.

\section{CONCLUSÕES}

Conclui-se a partir desta produção que o câncer de mama é uma doença ameaçadora de vida. Nesse sentido, o acompanhamento psicológico é de extrema importância para o manejo em todas as fases do adoecimento, desde o diagnóstico até a impossibilidade de cura. Considerando o paradigma biopsicossocial-espiritual ${ }^{12}$ e o princípio de integralidade do Sistema Único de Saúde (SUS) ${ }^{13}$, os pacientes devem ser assistidos por uma equipe multidisciplinar humanizada no cuidado ao paciente.

De acordo com a Política Nacional de Humanização ${ }^{14}$ (PNH), iniciativas de humanização do cuidado devem ser fortalecidas e o compartilhamento das práticas de gestão e atenção deve ser incentivado. 
Diante do exposto, o objetivo desta cartilha informativa foi disseminar o conhecimento acerca dos impactos psicológicos da neoplasia mamária para a população, através da educação em saúde. Além disso, foi possível incentivar a adesão às terapêuticas, a detecção precoce e o conhecimento sobre os direitos sociais. Ademais, fomentar os recursos disponíveis para o enfrentamento da doença e as potencialidades dos usuários e do SUS.

\section{REFERÊNCIAS}

1. Instituto Nacional de Câncer José Alencar Gomes da Silva. Ministério da Saúde. Controle do câncer de mama [artigo na internet]. 2021 maio [acessado 2021 jun 02]. Disponível em: <https://www.inca.gov.br/controle-do-cancer-de-mama/conceito-emagnitude>.

2. Instituto Nacional de Câncer José Alencar Gomes da Silva. Ministério da Saúde. Câncer de mama é preciso falar disso [cartilha na internet]. 2021 maio [acessado 2021 jun 02] Disponível em: 〈http://bvsms.saude.gov.br/bvs/publicacoes/cancer_mama_preciso_falar_disso.pdf $>$.

3. Secretaria de Estado de Saúde do Distrito Federal. Plano Distrital de Atenção Oncológica 2020-2023. 2020 [acessado 2021 jun 02]. Disponível em: <http://www.saude.df.gov.br/wp-conteudo/uploads/2018/02/Plano-Oncologico-formatadofinal.pdf $>$.

4. Instituto Oncoguia. Conheça os direitos das mulheres em relação ao câncer de mama [artigo na internet]. 2021 jun [acessado 2021 jun 02]. Disponível em:

<http://www.oncoguia.org.br/conteudo/conheca-os-direitos-das-mulheres-em-relacao-aocancer-de-mama/13006/42/>.

5. Fundação Laço Rosa. Tudo sobre os direitos dos pacientes com câncer de mama [cartilha na internet]. 2021 jun [acessado 2021 jun 02]. Disponível em: <. 
https://fundacaolacorosa.com/wp-content/uploads/2020/01/Tudo-sobre-os-direitos-dospacientes-com-c\%C3\%A2ncer-de-mama-1.pdf >.

6. Nogueira SP, Mendonça JV, Pasqualette HAP (2014). Câncer de mama em homens. Rev Bras Mastologia, 24(4), 109-114.

7. Gomes PA, Liberato R. Câncer de Mama: a relação da mulher com sua sexualidade após a mastectomia. In: Aguiar MAF, Gomes PA, Ulrich RA, Mantuani SB (2019). Psicooncologia: caminhos de cuidado. São Paulo: Summus, 2019. 394-403.

8. Venâncio JL (2004). Importância da atuação do psicólogo no tratamento de mulheres com câncer de mama. Rev bras de cancerologia, 50(1), 55-63.

9. SILVA LC (2008). Câncer de mama e sofrimento psicológico: aspectos relacionados ao feminino. Psicol. estud., Maringá, v. 13, n. 2, p. 231-237.

10. Ramos BF, Lustosa MA (2009). Câncer de mama feminino e psicologia. Rev da SBPH, 12(1), 85-97.

11. Vinogradov S, Yalom ID. (1992). Manual de psicoterapia de grupo.

12. Oliveira AD (2011). Psicologia da saúde e o paradigma biopsicossocial: um ensaio epistemológico. Rev. enferm. UFPE on line, 2309-2316.

13. Machado MFAS, Monteiro EMLM, Queiroz DT, Vieira NFC, Barroso MGT (2007). Integralidade, formação de saúde, educação em saúde e as propostas do SUS: uma revisão conceitual. Ciência \& saúde coletiva, 12, 335-342.

14. Brasil. Ministério da Saúde. Secretaria-Executiva. Núcleo Técnico da Política Nacional de Humanização. HumanizaSUS: Política Nacional de Humanização: a humanização como eixo norteador das práticas de atenção e gestão em todas as instâncias do SUS (2004) [acessado 2021 jun 02]. Disponível em: <http://bvsms.saude.gov.br/bvs/publicacoes/humanizasus_2004.pdf>. 